\title{
Design of an Airborne Three-Dimensional Separation Assistance Display
}

\author{
Joost Ellerbroek, Mark Visser, Stijn B. J. van Dam, Max Mulder, M. M. (René) van Paassen, Member, IEEE
}

\begin{abstract}
In the context of the NextGen and SESAR future airspace programmes, this paper describes a concept for an Airborne Separation Assurance (ASAS) display, that is designed to aid pilots in their task of self-separation, by visualizing the possibilities for conflict resolution that the airspace provides. This work is part of an ongoing research towards an ecological design of a separation assistance interface that can present all the relevant properties of the spatio-temporal separation problem. A work-domain analysis is described from which several perspective projections of traffic properties and travel constraints are derived. A display concept is proposed that presents heading and altitude action possibilities in a flight-path angle - track angle action space. Key issues in the current design are discussed, with recommendations for future work.
\end{abstract}

Index Terms-Ecological Interface Design (EID), Airborne Separation Assurance System (ASAS), self-separation, situation awareness

\section{INTRODUCTION}

I $\mathrm{N}$ today's airspace, increasing amounts of traffic are pushing the limits of capacity and safety. To facilitate continuing growth, new air-traffic management concepts are under development, which allow a more flexible use of airspace [1], [2]. These new concepts promote a shift towards airborne determination of user-preferred trajectories, where airspace capacity is expected to increase, while controller workload decreases. However, because the task of separation is shifted from the air traffic controller to the flight deck, it is expected that the pilot will need assistance to perform this task safely and efficiently.

The development of a support system requires a thorough analysis of what level of automation is required to meet with the overall system demands of safety, capacity and efficiency of flight. Crucial in this analysis will be the question of how these tasks should be allocated between humans and automation, and how the human actors can interact, and share their decision-making with the automation [3]-[5]. The interaction between automation and the human actor also requires transparent functioning of the automated system. The interface should provide operators with information regarding their own performance, as well as that of the automation, so that operators' self-confidence and trust correspond with

This work has been co-financed by the European Organisation for the Safety or Air Navigation (EUROCONTROL), under its Research Grant Scheme launched in 2008, and by the Nationaal Lucht- en Ruimtevaartlaboratorium (NLR). The content of the work does not necessarily reflect the official position of EUROCONTROL or the NLR on the matter.

The authors are with the Control and Simulation division of the Faculty of Aerospace Engineering, Delft University of Technology, 2629 HS Delft, The Netherlands. email: J.Ellerbroek@TUDelft.nl the capabilities of the system, and promote appropriate use of automation [6]-[8].

Although several display concepts have been developed as aids in the task of self-separation [9]-[13], most current research on Airborne Separation Assurance Systems (ASAS) focuses on the development of automated systems that assist pilots with the separation task [11], [14]-[17]. Generally, these systems provide the pilot with explicit, 'ready-to-use' resolutions to a separation conflict. Although they lead to lower pilot workload [18], these systems also hide the cognition behind the resolution advisory [19]. Without additional information, such systems may lead to low situation awareness (SA): the pilot is not fully aware anymore of exactly what is going on, and is unable to reason about the functioning of the automation, and other constraints and relationships within the environment under control [20]-[22]. This may lead to inappropriate reliance on the automation, which can have a significant impact on the overall levels of safety and efficiency. Under-reliance may lead to situations where the pilot prefers manual control over an automated (valid) resolution, leading to higher operator workload, and a possible reduction in performance and safety. On the other hand, over-reliance may cause pilots to use the automation in ways for which it was not designed, or to accept resolutions even when the automation is in error [6], [23].

In the air transport domain, lack of situation awareness is currently considered to be one of the the main causes for human error, responsible for at least seventy percent of the incidents and accidents that occur [24]. As airborne separation systems move towards more automation, it will become more important than ever that automation and instrumentation promote a high level of situation awareness. This leads to the fundamental question of 'what' needs to be presented to optimize human performance from the perspective of situation awareness [25]. In other words, how does one design for situation awareness? The work presented in this paper will employ a constraint-based approach, inspired by Ecological Interface Design (EID), a proven design paradigm from the domain of process control [26], [27], to design for SA [28], [29].

EID is a method that addresses the cognitive interaction between humans and complex socio-technical systems. Its approach to interface design gives priority to the workers environment, or 'ecology', focusing on how the environment poses constraints on the worker [28], [29]. Ecological displays are designed to allow for direct perception of the possibilities and constraints afforded by the work domain [30], [31]. This way, EID aims to support each level of cognitive control [32], 
while not forcing the operator to control at a higher level than necessary. By visualizing hidden constraints and relationships, ecological interfaces can transform what would otherwise be a cognitive task, into a perceptual task.

An ecological airborne separation assistance display then, should support pilot decision making in the task of selfseparation, rather than only providing an automated resolution. In the domain of process control, EID had the freedom to define a new interface for the operator. For air travel, however, pilots already make use of an existing, natural ecology (i.e., the ecology of locomotion). The key in designing an airborne separation support tool will be to not replace, but to enhance this existing ecology, by visualizing hidden affordances*, and exploiting the operator's natural adaptation to the ecology [34]-[39].

Preceding the design of an ecological display, a thorough analysis of the work domain is required, which should identify functionalities, constraints, and means-end relationships within that work domain. The main tools for this analysis are the Abstraction Hierarchy (AH), and the Skills, Rules, Knowledge taxonomy (SRK), both developed by Rasmussen [32], [40]. To make the transition from a workspace analysis to an effective interface design, functional visualizations of the affordances and constraints in the work domain need to be derived, so that they can be perceived and fluently transformed into goaldirected behavior, supporting the natural coupling between perception and action [41], [42].

This paper presents the results of a work-domain analysis of a self-separation airspace, and a concept for a separation assistance display. The analysis employs tools such as the Abstraction Hierarchy to obtain a clear image of how the work-domain shapes the affordances for a pilot in his task of self-separation. The display concept is the third in an ongoing design process towards an integrated, three-dimensional separation assistance interface, that presents an unambiguous, complete view of the airspace affordances, in an unmanaged traffic environment. The two previous concepts, the eXtended Airborne Trajectory Planning display (X-ATP) [38], and the Vertical Separation Assistance Display (VSAD) [39], presented maneuvering affordances in a heading-speed, and a flight-path angle (FPA) - speed action space, respectively. In a comparable fashion, the current concept will present maneuvering affordances in a FPA - track angle action space.

The paper is structured as follows: the following two sections will, respectively, present the results of a work-domain analysis, and illustrate the construction of functional visualisations of the constraints that followed from this analysis. The fourth section will introduce a concept of a separation assistance interface. The fifth section describes how the display concept can be used in more complex conflict situations. This paper concludes with a discussion on the key issues of the current display concept, with recommendations for future work.

\footnotetext{
* James J. Gibson defined affordances as opportunities for action, provided by an object or by the environment. An affordance is considered always in relation to the actor, and therefore dependent on the actor's capabilities [30], [33]. For instance, with respect to an engine, air affords propulsion, but with respect to a wing, air affords lift.
}

\section{WORK DOMAIN ANALYSiS FOR AIRBORnE SEPARATION}

The work domain under analysis in this study is limited to Trajectory-Based Operations (TBO) with self-separation. These operations involve trajectory (re-)planning on the flightdeck, in order to assure conflict-free flight in unmanaged, i.e., self-separated airspace. In the currently proposed future airspace concepts, the preferable mode of operation is one where automated 4D trajectory prediction and control are applied throughout the whole flight [2], [17], [43].

In this situation, the pilot's task will be one of monitoring separation, and selecting and applying resolution advisories, provided by the automation. The pilot should, however, be able to judge the fidelity of a proposed resolution, and be able to intervene in case the automation fails. Good situation awareness is therefore of paramount importance, and, in this study the Abstraction Hierarchy $(\mathrm{AH})$ will be employed to determine the relevant aspects of the work domain on several levels of abstraction. It will also serve to illustrate how the constraints and affordances on the different levels of abstraction interact with each other.

The abstraction hierarchy is a work-domain analysis tool that presents a stratified, hierarchical description of the workspace. Each stratum of the hierarchy represents the same system, but on a different level of abstraction. The levels are connected by means-end relationships between the adjacent levels. Along the vertical axis, commonly five levels represent the workspace at decreasing levels of abstraction, starting at the top with the purpose(s) for which the system was designed, all the way down to the spatial topology, properties, and appearance of the components that make up the system on the bottom level [40], [44]. In previous studies on a workspace analysis for the air transport domain, it showed that dividing the horizontal dimension of the AH between items "internal", and "external" to the ownship, provides a logical structure for an abstraction hierarchy that describes this domain [35], [36], [45].

\section{A. AH for airborne separation}

Fig. 1 shows an abstraction hierarchy for the workspace relevant to $\mathrm{TBO}$ and self-separation. In this hierarchy, the most relevant goals have been highlighted, along with the corresponding means-ends relationships. In the $\mathrm{AH}$, these goals are defined at the functional purpose level. In the case of ASAS self-separation applications, these are flying safely, productively, comfortably and efficiently through unmanaged airspace. For this analysis, it is assumed that safety can be assured by maintaining sufficient separation from potentially hazardous objects, such as other aircraft and terrain. In the current context, this means adhering to the defined separation minima between aircraft [46]. While they are relevant for assuring safe flight, issues such as staying within the flight envelope are kept out of the analysis. Although more complex in reality, in this paper it is defined that work is considered productive, as long as the distance to the destination is decreasing. For flight in general, comfort poses constraints such as upper limits on maneuver accelerations. The realization of 


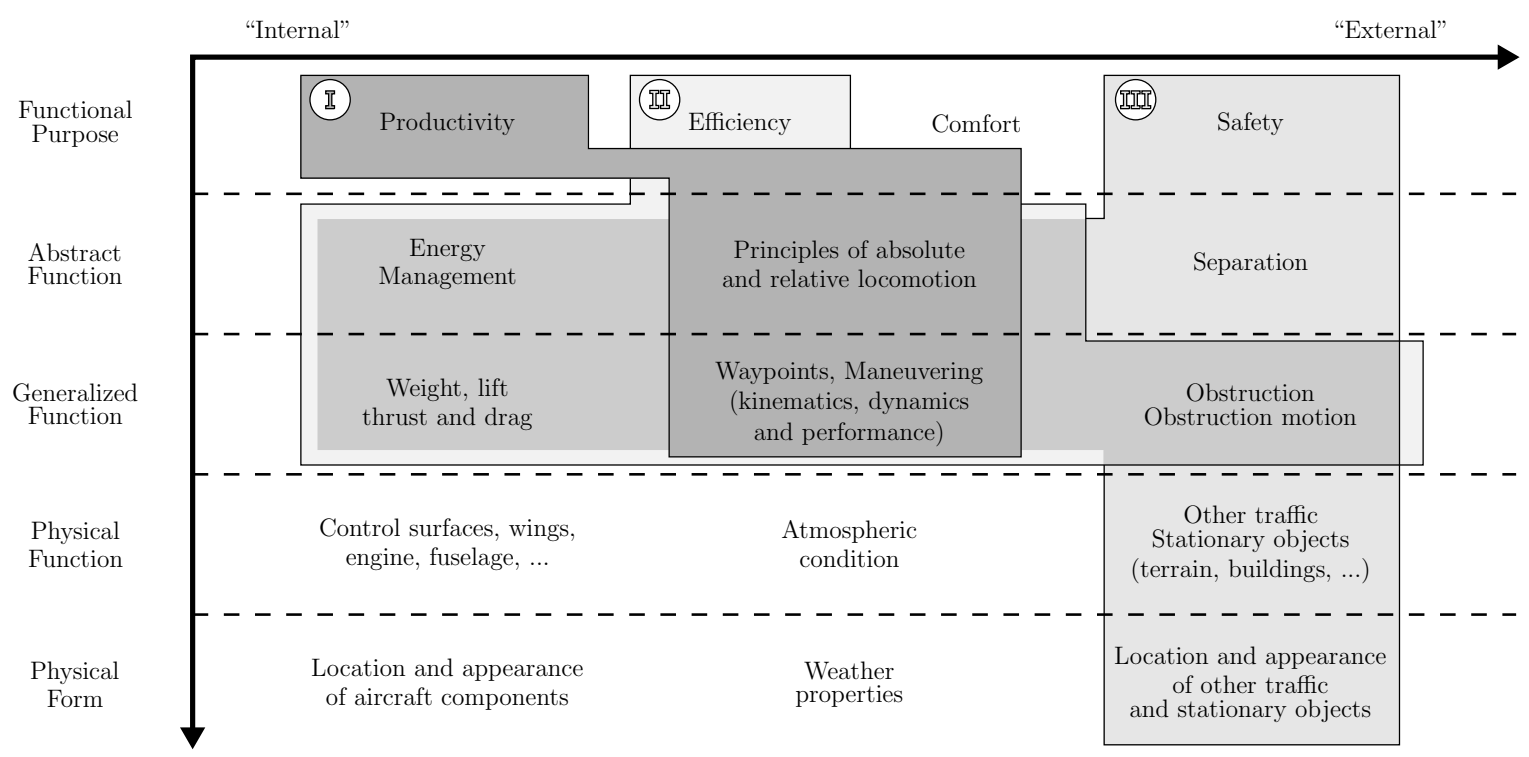

Fig. 1. Abstraction Hierarchy for the Separation Assistance Display. The analysis presented in this paper focuses on three functional goals. These goals, and the relevant means-ends relationships between levels have been highlighted in three groups in the hierarchy: (I) Productivity, (II) Efficiency, and (III) Safety.

efficiency is much more complicated, however, as it can be defined in many ways, such as fuel efficiency, or minimum path deviation. These constraints are beyond the scope of this paper.

The abstract function level describes the underlying relationships that govern the realization of the purposes of the system. In the case of air travel, this level contains the general physical laws that dictate flight, absolute and relative locomotion, and the geometrical properties of the separation problem [45]. Although for aircraft that follow a pre-defined, four-dimensional path, aircraft intent can influence the constraints that are derived on this level, the current study will only employ the current states to derive these constraints. Previous studies did incorporate the effect of intent on maneuver affordances [47], however, this is beyond the scope of the current study.

The generalized function level describes how the functions at the abstract function level are achieved, independent of the actual implementation of the system. Properties such as aircraft weight, lift, thrust and drag, and the maneuvering performance of the aircraft all impose internal constraints on the maneuver space of an aircraft. External obstructions further constrain this maneuver space, and dictate the (lack of) separation. On the bottom of the abstraction hierarchy, the physical form and functions are described by modeling the internal layout of aircraft components, and external airspace properties such as other traffic, weather, and terrain. The physical function level describes the various components, and their capabilities, and at the physical form level the appearance and location of components, the airspace, and other aircraft are described. The relevant internal and external constraints which can be derived from this abstraction hierarchy will be described in more detail below.

In this paper, the workspace content and boundaries are limited to trajectory planning functions in direct relation with conflict resolution and prevention during cruise flight. Functions related to aircraft control and stability, like staying within the flight envelope, and accounting for passenger comfort, are largely kept out of the analysis. The time horizon in which the workspace is analyzed is determined by the applicability of conflict management, and is approximately between 60 seconds and 10 to 20 minutes. Below 60 seconds, collision avoidance systems like the Traffic Collision Avoidance System II (TCAS II) must take over in order to prevent collision [48]. An upper threshold of 10 to 20 minutes is chosen because the vast majority of conflict resolution and recovery maneuvers take place in less than 20 minutes [17].

The scope of the current research is also restricted to the visualization of constraints that relate to tactical maneuvering, influences of ownship and intruder intent [47] are beyond the scope of this paper.

\section{B. Internal constraints}

The internal aircraft constraints that are relevant for this work domain analysis are mainly described on the abstract function and the generalized function levels of the work domain model. They relate to the various limitations on the performance of the aircraft, such as bank limits, turn dynamics, available engine power, stall, structural considerations, buffet characteristics, and requirements on emissions and passenger comfort. These limitations result in several constraints relevant to the task of trajectory planning, such as maximum turn rates, maximum and minimum operating speeds, fastest and steepest steady climb and descent, and the steepest steady climbing and descending turn.

Another important, although not directly perceivable constraining factor is the energy state of the aircraft: For an aircraft, speed and altitude share the same energy space. The mechanism that underlies the coordination of the controls, is the management of the aircraft's energy state (abstract function level, Fig. 1). Speed and altitude are directly related to the kinetic and potential energy of the aircraft. The total amount 
of energy is determined by the throttle, whereas the elevator is used to control the exchange of kinetic and potential energy. The total energy state of an aircraft essentially determines the affordances for maneuvering in terms of speed and altitude [34], [36]. Together, these internal constraints determine the part of the airspace that is reachable within a certain timespan.

\section{External constraints}

In unmanaged airspace, the reachable area that was defined by the internal aircraft constraints is further constrained by external factors, such as weather, terrain, other traffic, and the boundaries of the unmanaged airspace. In this analysis, the focus lies on the constraints imposed by other traffic. Traffic constraints are shaped by a minimum horizontal and vertical separation between any two aircraft, that should be adhered to at all times. With common values of 5 nautical miles horizontal, and 1,000 feet vertical separation, this results in a three-dimensional Protected Zone (PZ): A flat, threedimensional disc around each aircraft, that should remain clear of other traffic, see Fig. 2 [49], [50].

Intrusion of this space is referred to as a loss of separation. A conflict is defined as a future loss of separation, within a certain observation timespan (e.g., 5 minutes). In Fig. 3, a traffic conflict is illustrated from the perspective of ownship. This and subsequent figures depict a conflict situation between the ownship and one intruder aircraft. Although the principles presented in this analysis also hold for multiple intruder aircraft [38], this paper only uses single intruder conflict situations to illustrate the proposed concept, for the sake of clarity. In Fig. 3, the ownship is flying with velocity $\mathbf{V}_{\text {own }}$, and will eventually lose separation with the intruder aircraft, if no further action is taken. The point where separation is at a minimum is called the Closest Point of Approach (CPA). It can be seen that even when the ownship turns away from the conflict location, resulting in $\mathbf{V}_{\text {new }}$ in Fig. 3, separation can still be lost.

This adverse effect can be eliminated by examining the conflict situation in a relative velocity field [38], [39], [42]. Under the assumption that intruder and ownship state remain unchanged in the near future, a conflict can be predicted using the relative speed of ownship, with respect to the intruder aircraft:

$$
\mathbf{V}_{\text {rel }}=\mathbf{V}_{\text {own }}-\mathbf{V}_{\text {int }}
$$

Fig. 4 shows a conflict in the relative velocity field. When the line extended from the relative velocity vector crosses the intruder protected zone, a loss of separation will occur in the near future. By drawing lines through the ownship position,

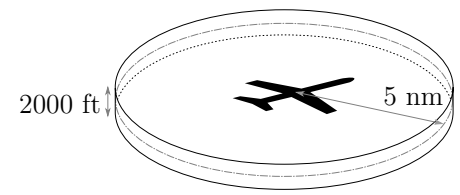

Fig. 2. The Protected Zone is defined as a disc-shaped area around each aircraft that should remain clear of other traffic.

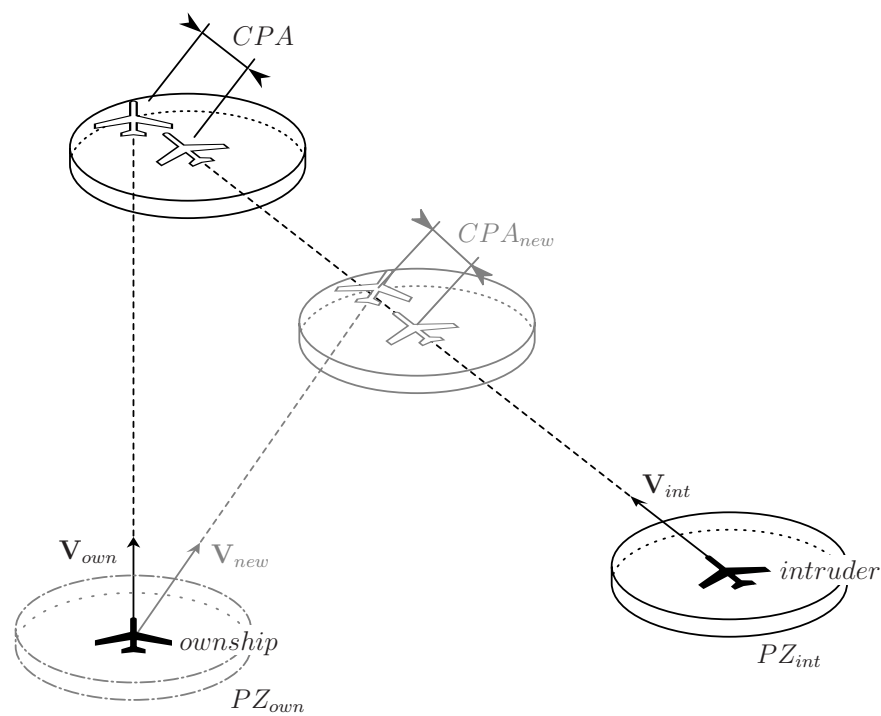

Fig. 3. A future loss of separation. It can be seen that even when ownship maneuvers around the area of the original conflict, separation can still be lost.

that are tangent to the intruder PZ, a three-dimensional wedgeshaped area can be defined, which marks the constraints that other traffic imposes on ownship relative motion with respect to an intruder aircraft (Fig. 4).

Unlike the absolute, spatial representation in Fig. 3, this representation only varies as a function of time (i.e., the wedge will expand as a function of the closing speed of the intruder aircraft, with respect to ownship). This means that for the current time, the three-dimensional wedge shape represents the complete set of relative velocity vectors that would result in a loss of separation.

\section{FUnCTIONAL PRESENTATION OF CONSTRAINTS}

Although a work-domain analysis provides insight in the structure and content of the work domain, it still requires a translation of this analysis into a practical interface design. Functional presentations of constraints and relations in a system should formulate the behavior of that system in terms that are relevant to achieving its ends. For trajectory planning, this implies that the goal-relevant affordances must be visualized in such a way, that the pilot's perception of these cues directly triggers desired goal-directed steering actions. A visualization is required that not only is compatible with the various identified constraints, but should also be able to reveal the relations between these constraints.

In the context of airborne separation, the behavior of the system is highly complex, as it is governed by the multi-

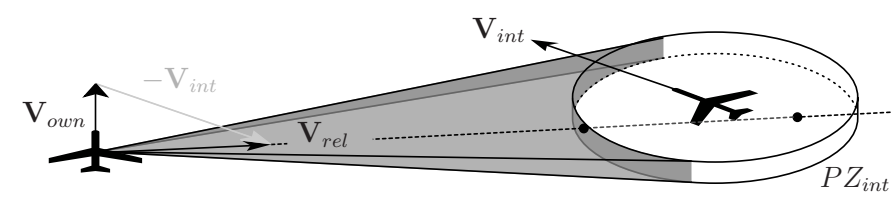

Fig. 4. A conflict situation expressed in a relative velocity field. Ownship and intruder are in conflict when the line that extends from the relative velocity vector crosses the intruder PZ. The wedge shape indicates the instantaneous set of constraints for the relative velocity vector. 
variable, non-linear dynamics of several aircraft, moving relative to each other. Because such a system has too many degrees of freedom to combine in a usable interface, a different description is required. This description should relate inputs that match common flight practice, to the goals and affordances of the system. In cruise flight, pilots control their aircraft by manipulating velocity, track angle, and altitude settings, using the autopilot, or by modifying the planned route in the Flight Management System. A successful separation assistance interface should relate these variables and their operational limits to the affordances of the airspace.

\section{A. Traffic constraints}

Fig. 3 already illustrated that presenting conflicts in absolute space is problematic: The closest point of approach is not constant, as it depends on the relative motion of two aircraft. It changes as a function of ownship and intruder velocity and heading. Therefore, steering away from a conflict based on the presentation in Fig. 3 will also move the CPA, and might not resolve the conflict at all. In other words, although a conflict presentation like the one in Fig. 3 provides the pilot with a visualization of the affordance of 'conflict', it does not show the affordance of 'avoidance', and is therefore an unsuitable conflict avoidance representation [38].

With the design of a horizontal and a vertical separation assistance display, previous studies illustrated that the affordance of avoidance can be consistently represented in a relative velocity field [38], [39] (Fig. 4). A disadvantageous aspect of the relative velocity field, however, is that it is hard for pilots to relate their affordances of control in absolute space, to a velocity constraint zone expressed in relative space. In previous research, van Dam [38] showed that this relation can be made visible, by translating the constraint zone and relative velocity vector by the intruder velocity vector, as shown in Fig. 5. This would be equivalent to adding $V_{\text {int }}$ on both sides of the equal sign in (1): the equation is still valid, but in the visualization (Fig. 5), the relation between the ownship velocity vector and the relative velocity constraint zone is made explicit.

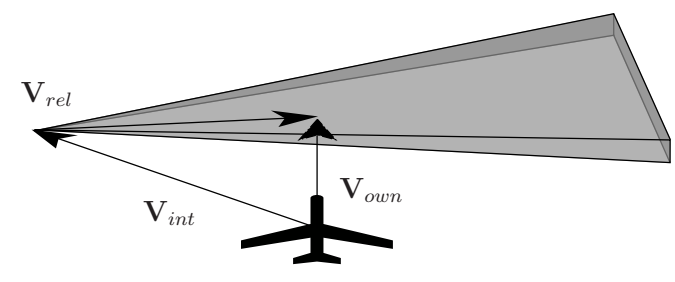

Fig. 5. Constraints on ownship velocity, expressed in an absolute velocity field. Ownship and intruder are in conflict when the tip of the ownship velocity vector is inside the three-dimensional constraint wedge.

The three-dimensional area in Fig. 5 represents the instantaneous constraints that the intruder position and velocity vector impose on the affordances for ownship locomotion (identifying the means-end relationship between obstruction on the generalized function level, and locomotion and separation on the abstract function level of the $\mathrm{AH}$ ). It not only reveals the individual affordances for ownship velocity, Flight-Path Angle
(FPA), and track angle, but also the interaction between the constraints of these three locomotion variables.

For the visualization of the three-dimensional workspace on a two-dimensional screen, two options can be distinguished: perspective displays and coplanar displays, each with their own benefits and drawbacks. A coplanar display corresponds more closely to the interfaces that are already present in the current flightdeck, whereas perspective displays have been found to have more "pictorial realism": they correspond more closely to the three-dimensional world [51], [52]. A drawback of coplanar displays in the current context is that some of the information on the interaction between locomotion constraints is lost, when these constraints are presented using separate horizontal and vertical projections. Perspective displays, on the other hand, suffer from perspective distortions, which can induce errors in judging distances on the display [53], [54]. The presentation of 3D structures such as the constraint zones also suffers from problems of occlusion: when viewed from a fixed angle, the front facing side of the structure hides the internal details of the structure.

Earlier designs of separation assistance displays reduced the complexity of the problem by relating several key controllable variables to a planar projection of the three-dimensional conflict situation. For instance, the X-ATP display presents the affordances for aircraft airspeed and track using a horizontal projection of the conflict situation [38], whereas the VSAD interface relates airspeed and vertical speed to a vertical projection of the constraints [39]. An often heard comment from professional airline pilots, in the evaluation of these previous designs, was, that while it featured as a valid and equal option in both displays, velocity changes are rarely used when resolving a conflict [50], [55]. Based on this feedback, this study investigates a cylindrical projection of an FPAtrack angle action space, which will be derived using spherical cutting planes based on constant velocity. The remainder of this section will discuss the applied projection method, and the derivation of affordance zones using the spherical cutting planes, respectively.

1) Projecting constraint areas onto a $2 D$ display: According to Gibson, a (human) observer perceives the threedimensional world as an optic array of solid visual angles, that correspond to distinct geometrical parts of the environment [30]. A perspective projection, such as the Synthetic Vision Display (SVD), can be regarded as a window (with a limited field of view) to this optic array. For such projections, the station point (the apex of the perspective projection) [53] would correspond to the point of observation, and horizontal and vertical coordinates in the projection correspond to the horizontal and vertical visual angles, respectively [37].

When the goal of the interface is to present the affordances of the complete surrounding airspace, the field of view of an SVD becomes a limiting factor. An ideal method should employ the egocentric natural perspective of an SVD, but in a way that is not restricted to its limited field of view. Several options can be considered. For instance, a section of the display could act as a 'rear-view-mirror', to visualize the environment behind the observer. This method, however, would essentially still result in a co-planar representation. 


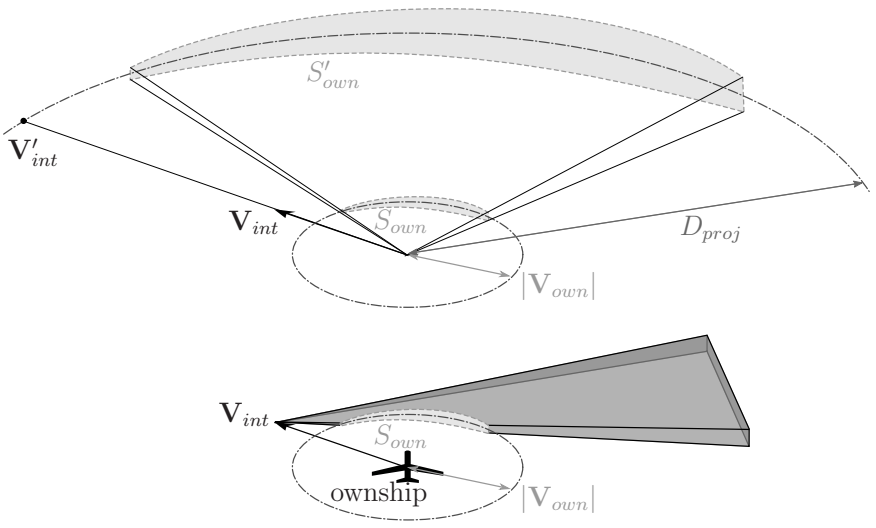

Fig. 6. The flight-path vector constraint at constant ownship velocity, $S_{\text {own }}$, is given by the intersection between a spherical shell, with a radius of constant ownship velocity, and the three-dimensional FPV constraint zone. Projection of this constraint area yields the visualization of the flight-path vector constraints, $S_{\text {own }}^{\prime}$.

The current concept will therefore use an approach, where the relation between the visual angles and the screen coordinates is defined using an equidistant cylindrical projection, with a horizontal visual angle range of $\phi \in\left[-180^{\circ}, 180^{\circ}\right]$, and a vertical visual angle range range of $\theta \in\left[-90^{\circ}, 90^{\circ}\right]$. This projection results in a single, continuous presentation of the entire surroundings, which directly relates horizontal and vertical visual angles, to horizontal $(x)$ and vertical $(y)$ screen coordinates, respectively:

$$
x=\theta, y=\phi
$$

This method of projection results in size and shape distortions for large vertical visual angles. However, the influence of this effect on the perception of the relevant combined internal and external constraints can be considered small, as the flightpath angle $\gamma$ will never be very large for commercial aircraft. For sake of clarity of the presented affordance cues, the line of sight of the projection is aligned with the current aircraft groundtrack, but is stationary with respect to roll and pitch angles of the aircraft, keeping vertical angle offset $\theta_{0}$ fixed. This results in an earth-referenced, 'outside-in' representation of the surroundings, as opposed to the more classical inside-out presentation employed in the current Primary Flight Display (PFD), and the SVD [56].

In the current concept, this projection method will be employed to project two affordance cues onto a widescreen display that presents the airspace affordances in an FPAtrack angle action space. The derivation of these cues will be described below. The first visualization provides the ownship flight-path vector constraints. The second visualization is constructed to inform the pilot on the geometry of each individual conflict. It visualizes relative intruder position and movement, and the urgency of a conflict. The derivations below will employ a virtual projection spherical surface, centered around ownship at an (arbitrary) distance, $D_{\text {proj }}$ (see e.g., Fig. 6), to illustrate the projections.

2) Ownship velocity direct constraints: The first projection is based on an intersection between the three-dimensional constraint zone (Fig. 5) and a spherical cutting plane with the current ownship velocity magnitude as radius, see Fig. 6. Based on pilot feedback that expressed a preference against the use of speed changes when resolving conflicts, it is assumed at this stage that velocity is kept constant. Future design iterations will investigate what exactly can be done in order to include a visualization of the effects of changes in ownship velocity.

Fig. 6 gives an example of how a spherical shell, with a radius of constant ownship velocity would intersect with the 3D constraint wedge from Fig. 5. The resulting area, $S_{\text {own }}$, is marked in light-grey in Fig. 6. The next step is then to project this constraint area on the perspective projection sphere described in the previous section, see Fig. 6. The resulting projection, $S_{\text {own }}^{\prime}$, shows the constraints for the ownship FlightPath Vector (FPV), that are imposed by the intruder's relative position and motion, for the current velocity of ownship. Note that the constraint areas from multiple intruder aircraft can be combined, in order to obtain a complete, instantaneous FPV action space, that addresses all current (possible) conflicts [38].

A display format was proposed by Gates et al. [57], that presents collision avoidance contours that resemble the flightpath vector avoidance contours in the current study. Although the contours presented here and the display presented by Gates et al look similar, they describe a different property of the conflict. The current study distinguishes between horizontal and vertical separation minima, and incorporates these in a visualization of flight-path vector affordances based on constant velocity. Gates' proposed format, however, does not directly consider visualization of affordances, but rather displays contours of equal (three-dimensional) Expected MissDistance (EMD).

Fig. 7 shows several shapes that the two-dimensional projection of the flight-path vector constraint area can take. Fig. 7(a) shows the result of the example projection from Figures 46. In this case, the intruder aircraft is situated to the right of ownship, flying at the same altitude. If ownship is flying level, ownship and intruder will eventually lose separation if no further action is taken. Fig. 7(b) shows a situation where the ownship velocity-sphere intersects the constraint-wedge twice. This can happen when an intruder is flying at a greater velocity than ownship, and intruder and ownship's tracks will be crossing sharply $\left(\Delta \psi \simeq 90^{\circ}\right)$. Fig. 7 (c) shows a situation where an intruder is overtaking ownship from directly behind, flying at a higher altitude. Because the intruder is close to ownship, almost all climbing maneuvers of ownship would lead to a loss of separation.

3) Conflict geometry projection: While the flight-pathvector constraint projection performs well at presenting constraints that the pilot can directly relate to the locomotion affordances of the aircraft, it does not show the specifics of each conflict: it is difficult to determine which intruder is causing a conflict, and how such a conflict would evolve. A second projection is constructed, therefore, to illustrate the geometry of individual conflicts. It combines a projection of intruder relative velocity constraints, and of the intruder protected zone, using the same projection surface as was used for the flight-path vector constraint zone.

The relative velocity constraints can be obtained using the intersection of a sphere, with its radius equal to the magnitude 
(a)

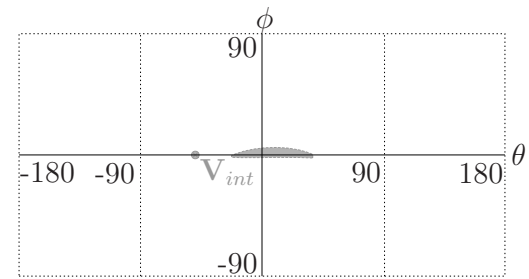

(b)

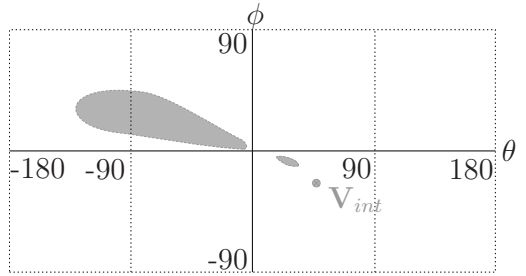

(c)

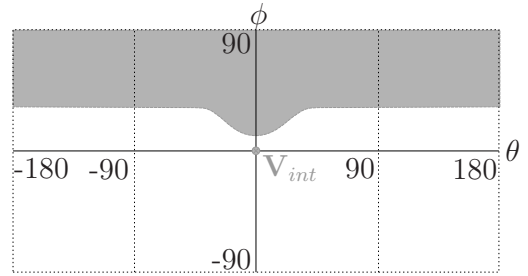

Fig. 7. Some examples of the projected flight-path vector constraint. (a): Basic example of a conflict, where the intruder crosses at an acute angle. (b): Velocity sphere intersecting wedge twice $\left(V_{\text {int }}>V_{\text {own }}\right)$. (c): Intruder overtaking ownship, at a higher flight level.

of the relative velocity vector $\mathbf{V}_{\text {rel,int }}=\mathbf{V}_{\text {int }}-\mathbf{V}_{\text {own }}$, and the three-dimensional wedge shape from Fig. 8. The resulting shape, indicated as $S_{\text {rel,int }}$ in Fig. 8, represents all velocities with equal magnitude of the intruder relative to ownship that correspond with possible future loss of separation. Next, this area is projected onto the projection sphere described in Sec. III-A1, together with the current relative speed, $\mathbf{V}_{\text {rel,int }}$, and the contour of the intruder PZ, see Fig. 8.

In this figure, $\mathbf{V}_{\text {rel,int }}^{\prime}$ is the projected relative velocity vector, $S_{\text {rel,int }}^{\prime}$ is the projection of the relative velocity constraint area, and $P Z_{\text {int }}^{\prime}$ is the projection of the protected zone of the intruder. In the two-dimensional presentation on the display, the location on the display of each of these combined projections illustrates the direction of the line-of-sight to the respective intruder. The altitude difference between ownship and the intruder is further emphasized by the curvature of the projected intruder PZ. The curvature in this projection is caused by the circular shape of the protected zone, and changes as a function of the vertical position of the intruder, relative to the ownship. Together with the projection of the

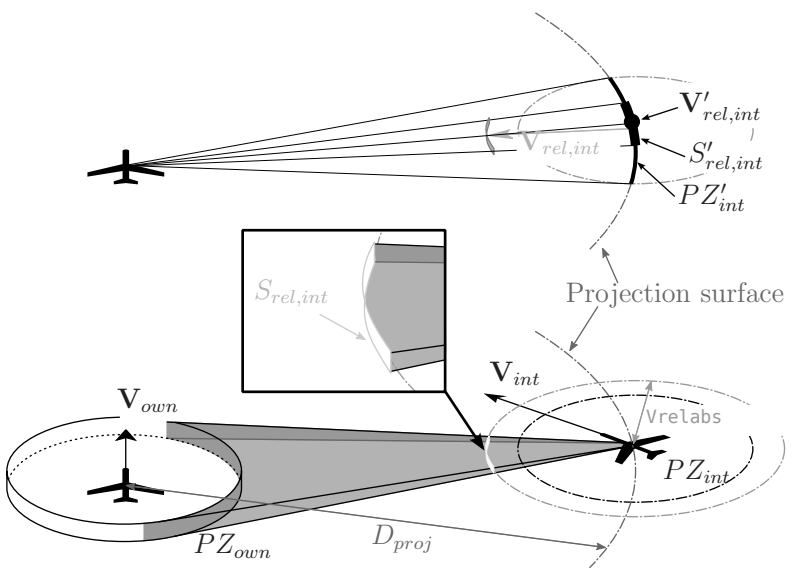

Fig. 8. Projection of the relative intruder velocity constraints $\left(S_{\text {rel,int }}^{\prime}\right)$, the relative intruder velocity vector $\left(\mathbf{V}_{\text {rel,int }}^{\prime}\right)$, and of the protected zone geometry $\left(P Z_{\text {int }}^{\prime}\right)$, result in the visualization of the 'puck'.

relative velocity vector, $\mathbf{V}_{\text {rel,int }}^{\prime}$, the constraint area $S_{\text {rel,int }}^{\prime}$ gives an indication of how a possible conflict would evolve. If the projection of the relative speed vector lies within constraint area $S_{\text {rel,int }}^{\prime}$, the intruder and ownship are in conflict. The direction of $\mathbf{V}_{\text {rel,int }}^{\prime}$ indicates how ownship and the intruder will pass each other, whereas its proximity to the boundary of the constraint area shows how closely they will pass each other. Furthermore, with respect to the size of the intruder's projected $\mathrm{PZ}$, the size of the relative velocity constraint zone can be used as an indication for the closing rate between intruder and ownship.

As a result of the projection onto the projection sphere of the relative velocity vector, it is no longer distinguisable whether $\mathbf{V}_{\text {rel,int }}$ is aimed towards, or away from ownship. To resolve this ambiguity on the display, four lines are drawn from the boundaries of the puck towards the tip of the relative velocity vector, when $\mathbf{V}_{\text {rel,int }}$ is aimed towards ownship. These lines are absent when $\mathbf{V}_{\text {rel,int }}$ is aimed away from ownship.

The two-dimensional presentation of this projection results in a (ice-hockey) puck-like shape. Fig. 9 shows two examples of what this 'puck' may look like, for different situations. In Fig. 9(a), an intruder is flying in front of, and to the right of ownship, at a higher altitude than ownship. The projected relative velocity vector points outside of the relative velocity constraint zone, indicating that ownship and intruder are not in conflict. The direction of the relative velocity vector reveals that the intruder will eventually pass behind and above ownship, if neither aircraft maneuvers.

In Fig. 9(b), the intruder is flying behind ownship, on the same course, but at a higher velocity. The relative velocity vector is such that it points directly at ownship, and therefore is located in the center of the 'puck'. This means that in this situation a collision would occur, if no further action is taken. Note that when both the ownship as well as the intruder aircraft are equipped with the same interface, their representations would be very similar: the appearance of the 'puck' would be identical, only at a different location on the screen.

Note that the current derivations of the puck and the flightpath vector avoidance zone assume instant state changes. It can be shown that this is a safe assumption when a predicted conflict is still in the far future [58], [59]. However, maneuver dynamics will start to play a larger role when conflicts become more imminent: in the case of tactical maneuvers (within 10 minutes of a predicted conflict), unmodeled dynamics will cause significant errors, particularly speed maneuvers [58]. To compensate for these inaccuracies, future iterations of the interface concept will incorporate maneuver dynamics in the presentation of airspace affordances.

\section{B. Production and maneuvering constraints}

For the horizontal X-ATP concept, productivity was considered in terms of destination approximation, which for the horizontal projection in the X-ATP display translated into relative track angle constraints of $\pm 90^{\circ}$ around the desired 
(a)

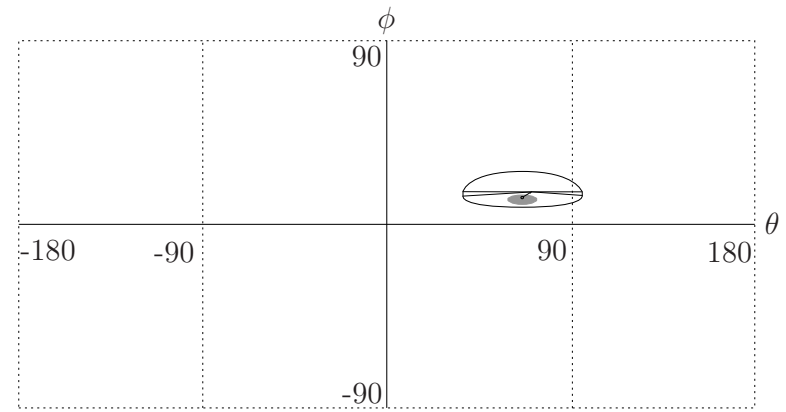

(b)

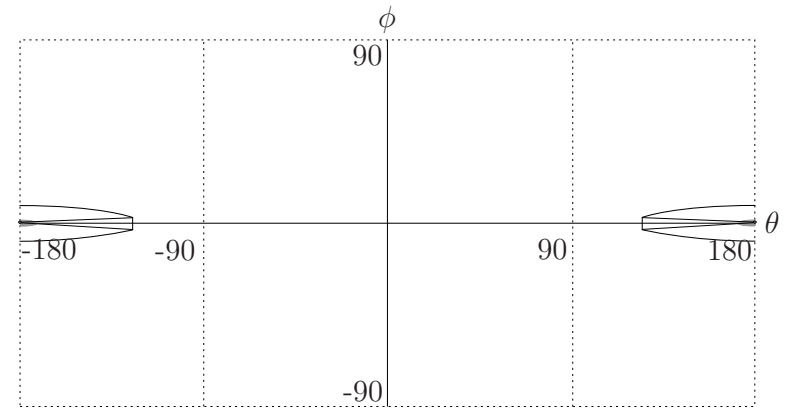

Fig. 9. Some examples of the 'puck'. (a): An intruder passing safely behind and over ownship. (b): An intruder overtaking ownship.

track [38]. For the current concept these limits can also be marked on the display. It is further assumed that altitude change resolutions do not affect the production goal, as the horizontal trajectory is maintained, and delays caused by vertical maneuvers are minimal.

The constraints for vertical maneuvering have been described intensively in [37]. For the current application, the climb and glide performance are considered at a constant airspeed. While potential - kinetic energy exchanges might indeed form interesting maneuvering possibilities for conflict resolution, they are beyond the scope of the current study.

Climbing and gliding constraints at a constant airspeed can be determined as a function of the maximum thrust, the glide ratio (or aerodynamic efficiency), and the roll angle. At a given altitude, these constraints translate into a maximum and a minimum flight-path angle $\gamma$. In an angular projection such as described in Sec. III-A1, these constraints can be indicated with lines of constant vertical visual angle.

In order to perform a steady climb or descent, a pilot has to manage the energy state of the aircraft. More precisely, he/she has to keep the kinetic energy rate to zero, and the potential energy such, that it matches the desired flight-path angle. In previous work, Amelink [34] described how the total energy rate of an aircraft can be expressed as a total energy angle, $\gamma_{E}$. When shown in conjunction with the flight-path angle, $\gamma$, the total energy angle also reveals the individual kinetic and potential energy rates to the pilot.

\section{The Display CONCEPT}

Figure 10 illustrates the first design prototype of the separation assurance interface, which presents the separation assistance display elements introduced in the previous section, on an equidistant cylindrical projection of the airspace surrounding ownship.

\section{A. Elements of the display}

In this concept, the horizontal axis represents the full track angle range, \pm 180 degrees, and behaves like a compass. This means that when ownship changes heading, the separation elements on the display shift horizontally, corresponding to the change in heading. The production constraints from the work-domain analysis are visualized by indicating the \pm 90 degree limits with vertical lines (1. The vertical axis of the current concept presents the vertical visual angle, in an earthfixed frame of reference, ranging from -90 to 90 degrees.

Vertical ownship maneuvers are visualized with the vertical offset of the flight-path vector symbol 2, from the center-line of the display. Together with the flight-path angle, the energy angle is shown as well 3, i.e., the flight-path the pilot can select to realize a steady climb or descent. The steepest steady climb and descent for the current velocity are shown with the dashed lines 4. Together, they relate to the function of energy management, and with the constraints they relate to the safety goal.

Intruder aircraft that are in conflict with ownship, or can get into conflict with ownship within the prediction horizon, are shown using the puck 5 . For each intruder aircraft within detection range, one puck is shown on the interface. The center of the puck represents the line of sight to the intruder. The arrow and its four lines indicate the direction and (projected) magnitude of the relative velocity of the intruder. When the lines are present the intruder is moving towards ownship, when they are absent the intruder is moving away from ownship. The size of the puck depends on the distance to the ownship (smaller is further away).

The shaded area in the puck represents the constraints for the velocity of the intruder, relative to ownship. If the tip of the relative velocity vector is located inside this area, and has the four lines attached, a loss of separation will occur within the look-ahead horizon. The puck primarily relates to the safety goal, and shows relations between obstruction (motion), relative motion, and separation from the $\mathrm{AH}$.

Constraints on ownship flight-path vector are shown with a shaded area 6 . Note that this area is only valid for the current speed. Conflict urgency can be indicated by (locally) varying the color of the flight-path constraint area. In case of multiple intruders, constraint areas can be combined. In situations where areas with different conflict urgency levels overlap, the color of the shaded area is determined based on the highest urgency. 6 shows the affordance of conflict, as well as the affordance of avoidance, and relates to the safety goal, as well as to the efficiency goal (through the shortest way out principle [38]). Intruder flight-path vectors are shown as dots on the display $\boldsymbol{7}$. Moving the ownship flight-path vector towards one of these dots to resolve a conflict will lead to a very inefficient resolution, as this maneuver will cause ownship to fly parallel to the intruder [38]. 


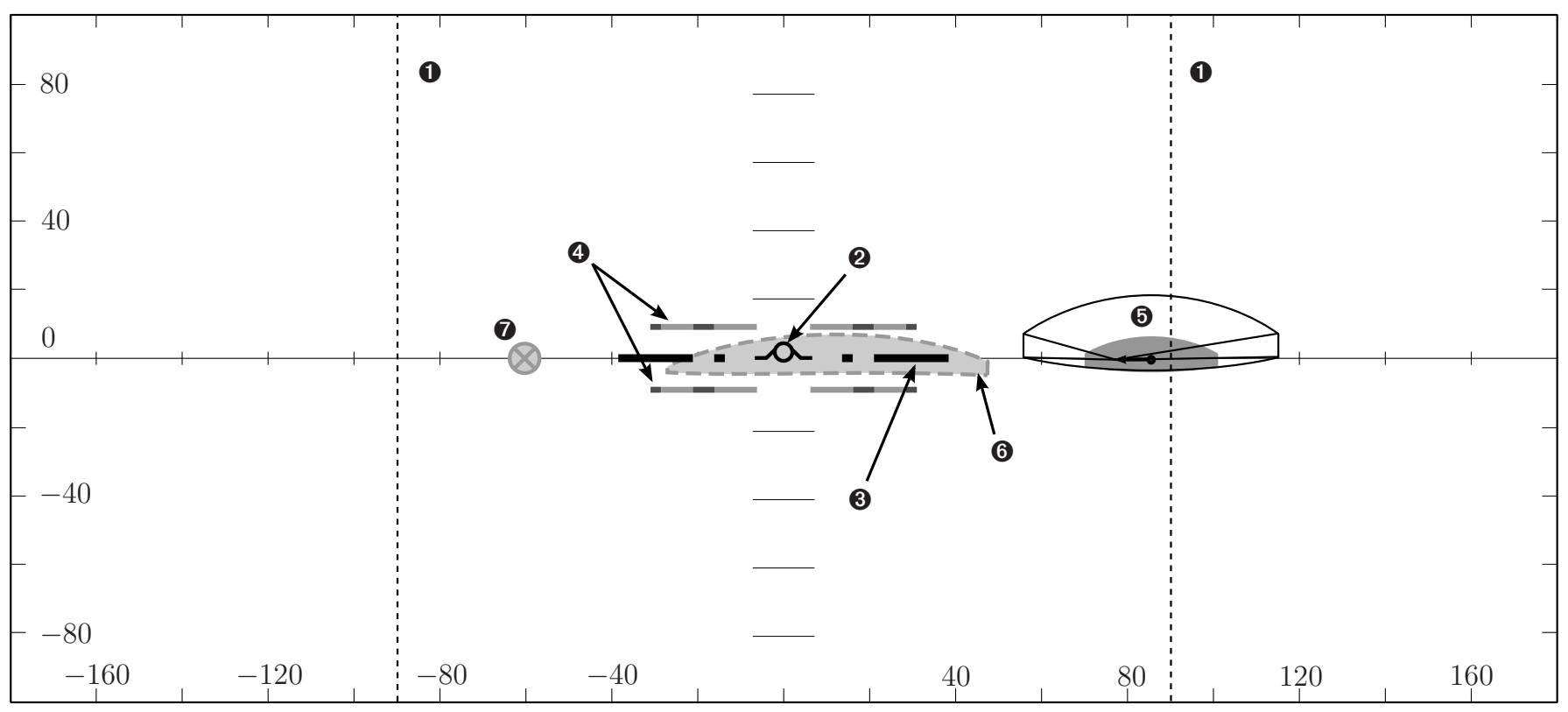

Fig. 10. The interface design concept showing the example situation. The separation assistance elements numbered in this figure are: $\mathbf{0}:$ heading production constraints, (2: flight-path vector, (3: energy angle, (4: steepest steady climb/descent, 6: the 'puck', 6: FPV constraint zone, and $\mathbf{0}$ : intruder flight-path vector.

\section{B. Dynamic behavior of the display}

Because the display projections depend not only on relative speed, but also on relative position of the intruder aircraft, the projection elements will change their shape over time, even when no corrective action is performed to resolve a conflict. Consequently, different conflict geometries will result in a different emergence of shapes. It is expected that after prolonged use of such a display, pilots will recognise patterns in this behavior. These patterns become familiar cues that can trigger rule-based actions [32].

Fig. 11 shows an example dynamic scenario in planview, where one intruder aircraft passes behind ownship. In the example, ownship is flying level, heading north, at an altitude of $20,000 \mathrm{ft}$, with a groundspeed of $390 \mathrm{kts}$. The intruder is initially flying at $36,000 \mathrm{ft}$, but descending with a vertical speed of $2000 \mathrm{ft} / \mathrm{min}$, and a groundspeed of $525 \mathrm{kts}$. Initially, the intruder is flying just behind, and east of ownship, with a track angle of $330^{\circ}$, at a distance of $36 \mathrm{NM}$. The resulting $\mathbf{V}_{\text {rel, int }}$ is directed just behind ownship, resulting in the intruder passing behind ownship during the course of the scenario.

Fig. 12 illustrates the emerging behavior of the flight-path constraint area in the example scenario. Fig. 12(a) shows the situation at $t=0$. The intruder is illustrated with the puck, and the constraint area for the ownship Flight-Path Vector (FPV) is split into two parts. Both FPV constraint areas grow over time, up to the point that the larger of the two is stretched over the sides of the interface, as illustrated in Fig. 12(b)(c). In Fig. 12(d), the larger area 'opens up', and stretches over the whole top of the display. In Fig. 12(d), the small and large constraint areas start to grow towards each other, until they merge in Fig. 12(e). After that, the stretched area shrinks again and becomes smaller, up to the point where it finally disappears (Fig. 12(j)), i.e., there is no possibility left to get into conflict with the intruder, at the current velocity.

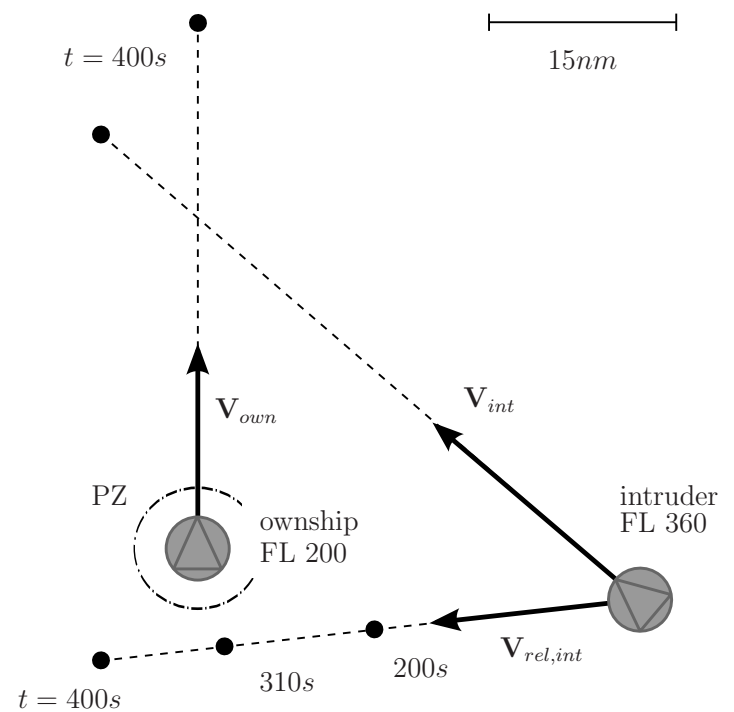

Fig. 11. Planview of example dynamic scenario.

\section{Practical Application}

The appropriateness of the concept for real-world applications depends on how well the proposed constraint projections extend to complex situations. Scenario's such as multiple intruder conflicts, and complex trajectories will ultimately determine the succes of the concept. This section describes how the current concept behaves in more complex situations, and possibilities for extending the concept.

When multiple conflicts occur simultaneously, each of the conflicting aircraft would be represented on the display with its own 'puck', and corresponding FPV constraint area. Because each constraint area represents a set of state vectors that result in a conflict with that aircraft, a combination of conflict areas from multiple aircraft, superimposed onto each other, 
(a) $t=0 \mathrm{~s}$

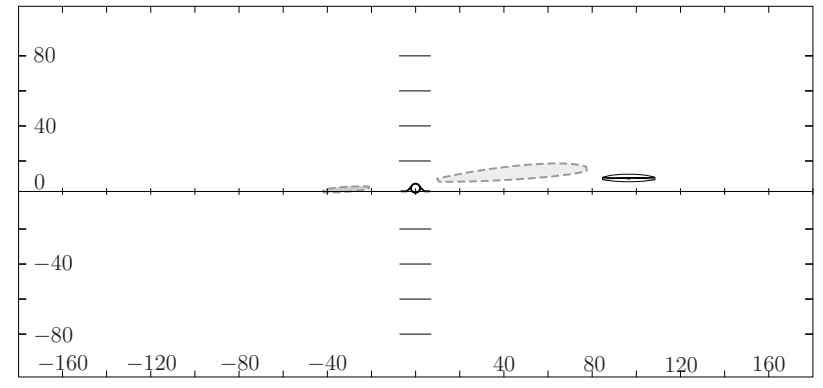

(c) $t=230 \mathrm{~s}$

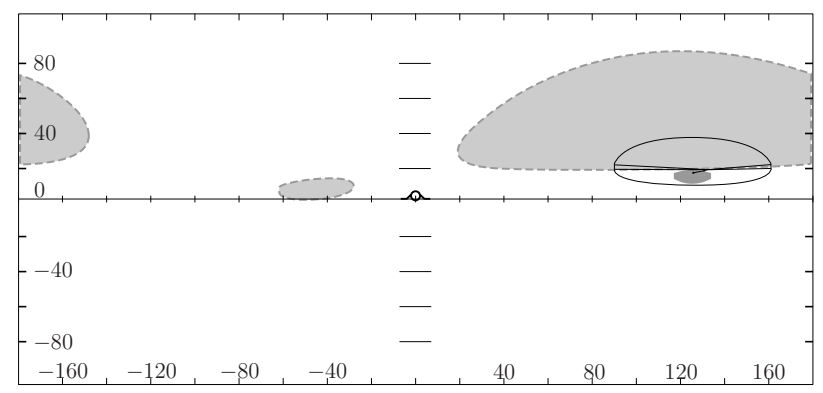

(e) $t=270 \mathrm{~s}$

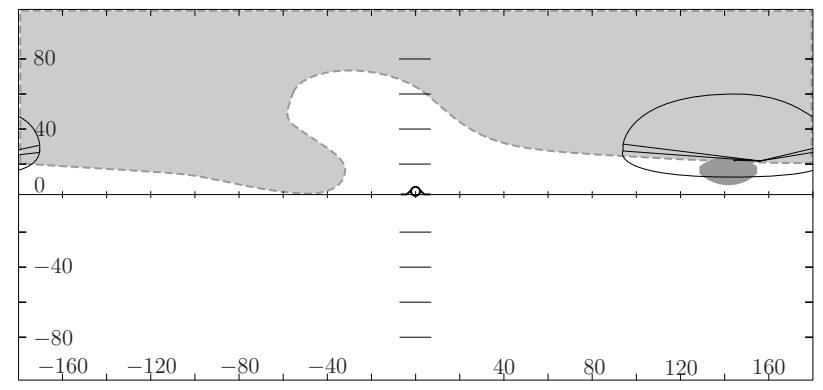

(g) $t=325 \mathrm{~s}$

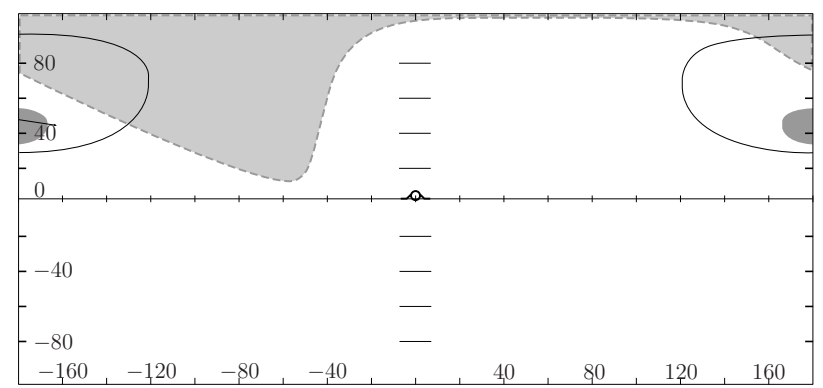

(i) $t=360 \mathrm{~s}$

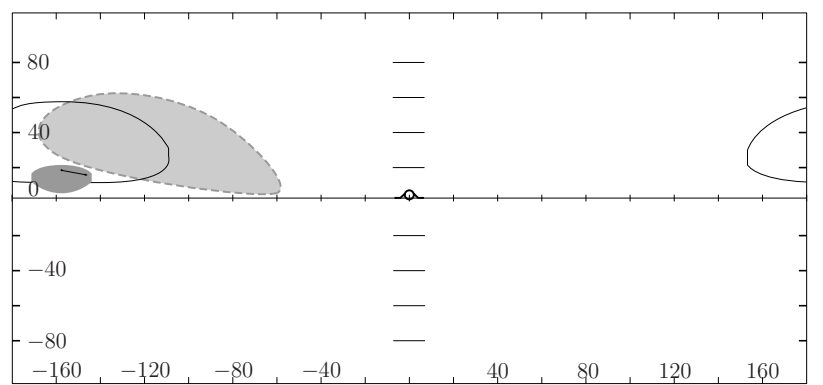

(b) $t=200 \mathrm{~s}$

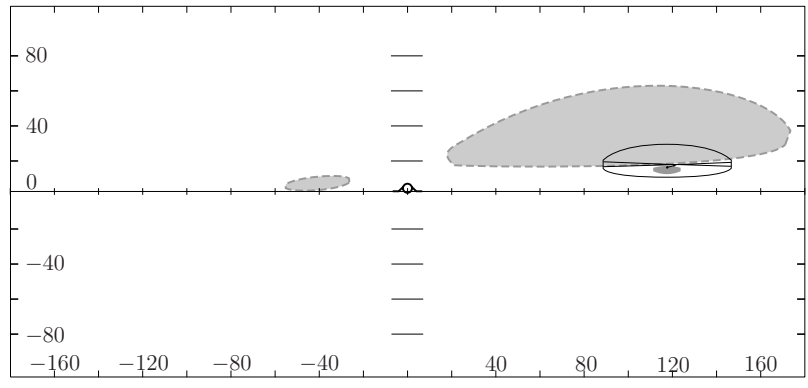

(d) $t=250 \mathrm{~s}$

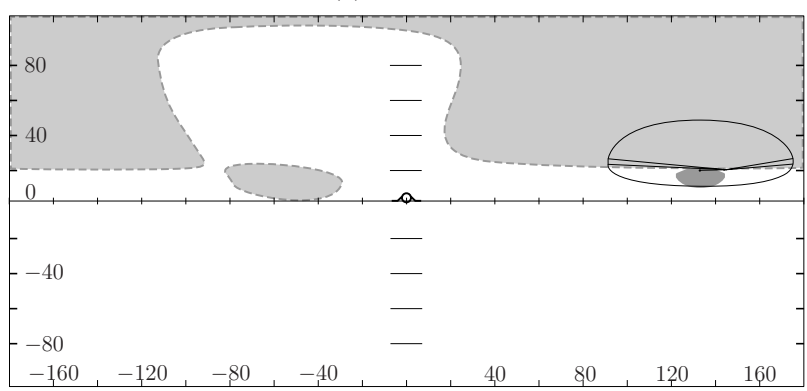

(f) $t=310 \mathrm{~s}$

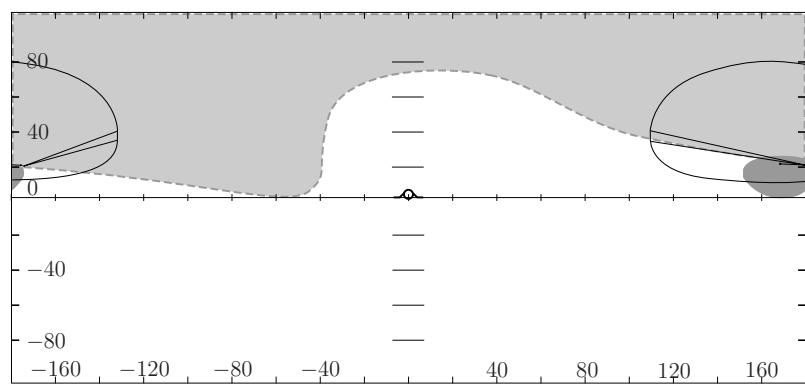

(h) $t=340 \mathrm{~s}$

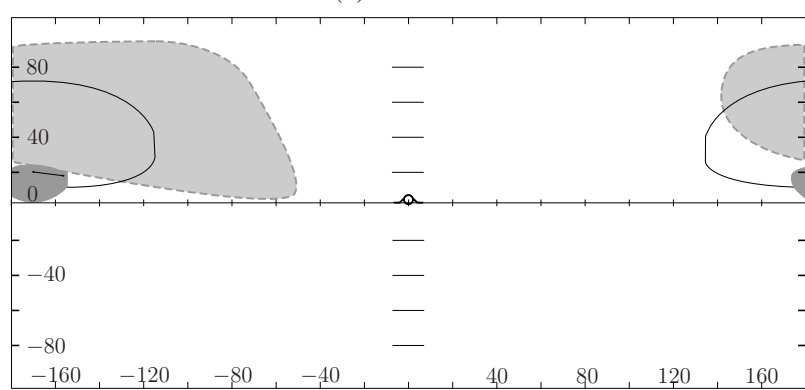

(j) $t=400 \mathrm{~s}$

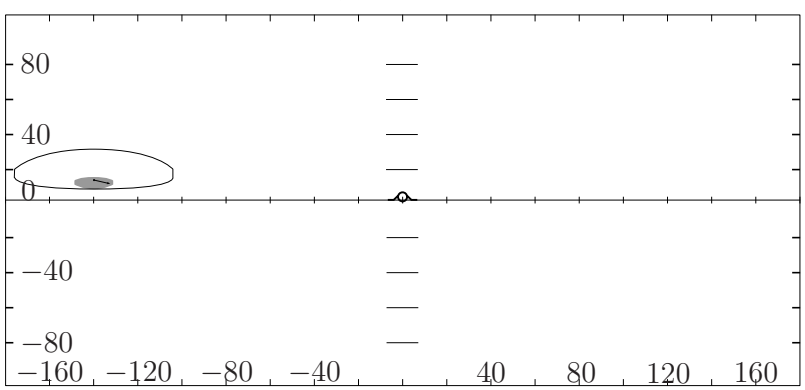

Fig. 12. Example of a descending intruder aircraft passing behind ownship at distinct times. 
represents the set of states that would lead to a conflict with one or more of these aircraft. As a result, any state outside of this combined constraint area is a solution to all of the current conflicts. When such a global solution is unavailable or undesirable, conflicts will have to be solved in sequence.

This, in turn, requires the operator to determine an appropriate sequence in which to solve the conflicts, introducing the need to visualize the priority of each conflict. Color coding of the flight-path vector constraint area can be used to indicate time to loss of separation for a particular conflict. Two options are available: the first option gives a single color to a constraint area belonging to a certain intruder aircraft, where the color corresponds to the time to LoS with the current state vector. A second option would make use of the fact that every point in a constraint area corresponds to a certain state vector, which in turn corresponds to a certain time to loss of separation. This way, each point in a conflict area would be colored individually. The color of an individual constraint area then communicates the priority of the corresponding conflict.

The current approach of presenting the airspace affordances, i.e., presenting them as constraints on the current state, has several benefits: it shows a clear picture of how traffic influences the pilot's maneuvering possibilities (in terms of possible, conflict-free states), and it can be used to create combined visualizations for multiple state dimensions (e.g. the X-ATP display showing combined heading/speed affordances, the VSAD FPA/speed, and the current concept presenting combined FPA/heading affordances). These displays support the pilot on Endsley's first two levels of SA (perceiving situational elements, and comprehending the meaning of the perceived variables, and their significance with regard to the system goals), and to a certain extent also on the third level of SA (projecting the current state, or a target state into the future) [60]. When intent (ownship and intruder) is taken into account, however, projection is no longer a trivial extrapolation in time of the aircraft state vector. Instead, the affordance space changes as a function of space and time due to Trajectory Change Points (TCP), and other changes of state or intent.

Van Dam already showed for the horizontal case, that the dimension along the bisector of the triangular forbidden area determines the time at which the closest point of approach with the respective intruder will occur [47], with the triangle origin representing $t_{C P A}=\infty$. Using this property, the part of the triangle where $t_{C P A}>t_{T C P}$ can be discarded. Extrapolations of the future track can be used to show constraints due to intruder state beyond its TCP. A similar method can be used to include intent in the current concept.

\section{DISCUSSION}

The work presented in this paper is part of an ongoing study on the design of a trajectory planning aid. The intended goal is to obtain a graphical interface, that supports pilots in their new task of airborne reconfiguration of a pre-planned trajectory, in case of traffic conflicts in unmanaged airspace. For a pilot to function consistently well in this new task, the interface should promote a high level of situation awareness, supporting the pilot in routine, as well as unforeseen situations.
This study adopts an EID-inspired, constraint-based approach, where results from a work-domain analysis on multiple levels of abstraction are used to develop a visual representation of the travel constraints.

To improve situation awareness, and support appropriate trust in, and interaction with automated systems, it is necessary for the automation to be transparent and understandable to the operator. An ecological interface tries to achieve this by revealing more about the functioning of the work domain [61]. The current interface concept tries to realize this by showing the implications of other traffic for the affordances of locomotion in relative space, and how they relate to ownship performance constraints in absolute space. When used in combination with an automated system that provides explicit resolutions, the display helps improve operator trust and understanding of an automated resolution, by helping him understand how constraints shape possible resolutions.

Previous work from this study resulted in two concepts of separation assistance displays: the X-ATP display presents a horizontal projection of the constraints and affordances, which resulted in a speed-track angle action space overlay on the Navigation Display (ND) [38]. This display has since then been extended, by showing the effects of maneuver dynamics and ownship and traffic intent on the presentation of the affordance space [47], [59]. The VSAD display works in a similar fashion, but instead of showing a horizontal projection of the locomotion constraints, it uses a vertical constraint projection to create an FPA-speed action space that is presented on a Vertical Situation Display (VSD) [39].

Both these displays provide a projection of a certain action space. The action, here, is the action of locomotion of an aircraft in three-dimensional space, which can be defined by flight parameters track angle, flight-path angle, and velocity. For this three-dimensional action space, the X-ATP and VSAD displays can be regarded as two orthogonal views on the threedimensional affordance space. The FPA-track angle affordance zone presented in this paper, then, would be the remaining third view. Considering the preference of airline pilots to keep the airspeed constant [50], [55], it can be hypothesized that the FPA-track angle combination should be the preffered option of these three projections. This should be addressed in a comparative experiment.

One of the main design challenges, is to make use of an aircraft pilot's existing ecology. For travel planning and avoidance, pilots already make use of the outside view and existing cockpit instruments, to perceive the affordances of the airspace. The challenge is therefore not to replace, but to enhance this perception. One way to do this is to find a compatible display to adapt with the separation assistance elements. This method is employed in the previous concepts: the $\mathrm{X}$-ATP display used the ND to present the travel affordances, and the VSAD projected the situation on the VSD.

The current concept would be most compatible with the Primary Flight Display (PFD), since they both present their information in the track angle / flight-path angle space. However, a problem with this combination is that a PFD presents its information in an "inside-out" fashion (i.e., the aircraft symbol is stationary, whereas the horizon moves). This is an 
unfavorable situation when separation avoidance elements are shown on this display. Because these elements are related to the orientation of the ownship, they will move and rotate with the horizon, making them more difficult to interpret. Other options would, then, be auxiliary displays such as the Electronic Flight Bag (EFB).

A consequence of the current choice of cutting-planes is that the visualized affordances relate to ownship motion in terms of track angle and vertical speed changes, however, they do not show the affordances in terms of ownship velocity. Although pilot feedback in the evaluation experiments of the previous concepts already indicated that velocity changes are not preferred, and therefore rarely used when resolving conflicts, the 'perfect ecological interface' would ideally present the affordances for all of the pilot's maneuvering options. Also, speed changes might be required to satisfy other goals than safety, for instance to compensate for time deviation along track, to adhere to time constraints at the next waypoint. In terms of requirements following from the efficiency goal, a pilot may for instance also consider a constant-throttle vertical maneuver, where velocity cannot be assumed constant.

It is clear that the step from work domain analysis to display concept is far from a trivial one, and more than one iteration between analysis and design will be required to work towards a fully functional, and mature design [45]. In these iterations, concessions are unavoidable when trying to present the behavior of a four-dimensional work-domain on a twodimensional surface.

\section{REFERENCES}

[1] JPDO, "Concept of Operations for the Next Generation Air Transportation System, Version 2.0," Joint Planning and Development Office, Tech. Rep., Jun. 2007.

[2] SESAR Consortium, "SESAR Definition Phase D3: The ATM Target Concept," Eurocontrol, Tech. Rep. DLM-0612-001-02-00, 2007.

[3] D. A. Norman, "The "Problem" of Automation: Inappropriate Feedback and Interaction, not "Over-Automation"," Philosophical Transactions of the Royal Society of London, vol. 327, no. 1241, pp. 585-593, Apr. 1990.

[4] N. B. Sarter and D. D. Woods, "Pilot Interaction With Cockpit Automation: Operational Experiences With the Flight Management System," The International Journal of Aviation Psychology, vol. 2, no. 4, pp. 303-321, 1992.

[5] G. Lintern, "An Affordance-Based Perspective on Human-Machine Interface Design," Ecological Psychology, vol. 12, no. 1, pp. 65-69, 2000.

[6] J. D. Lee and N. Moray, "Trust, Control Strategies and Allocation of Function in Human-Machine Systems," Ergonomics, vol. 31, no. 10, pp. 1243-1270, 1992.

[7] —, "Trust, Self-Confidence, and Operators' Adaptation to Automation," International Journal of Human-Computer Studies, vol. 40, no. 1, pp. 153-184, 1994.

[8] B. M. Muir, "Trust in Automation. 1: Theoretical Issues in the Study of Trust and Human Intervention in Automated Systems," Ergonomics, vol. 37, no. 11, pp. 1905-1922, 1994.

[9] C. Meckiff and P. Gibbs, "PHARE Highly Interactive Problem Solver," Eurocontrol, Tech. Rep. 273/94, Nov. 1994.

[10] R. Azuma, H. Neely, M. Daily, and M. Correa, "Visualization of Conflicts and Resolutions in a "Free Flight" Scenario," in Proceedings of IEEE Visualization, 1999, pp. 433-436.

[11] J. M. Hoekstra, R. N. H. W. van Gent, and R. C. J. Ruigrok, "Designing for Safety: the Free Flight Air Traffic Management Concept," Reliability Engineering and System Safety, vol. 75, pp. 215-232, 2002.

[12] R. Canton, M. Refai, W. W. Johnson, and V. Battiste, "Development and Integration of Human-Centered Conflict Detection and Resolution Tools for Airborne Autonomous Operations," in International Symposium on Aviation Psychology, 2005, pp. 115-120.
[13] W. R. Knecht, "Testing a Multidimensional Nonveridical Aircraft Collision Avoidance System," Human Factors, vol. 50, no. 4, pp. 565-575, 2008.

[14] J. K. Kuchar and L. C. Yang, "A Review of Conflict Detection and Resolution Modelling Methods," IEEE Transactions on Intelligent Transportation Systems, vol. 1, no. 4, pp. 179-189, 2000.

[15] R. Ghosh and C. J. Tomlin, "Maneuver Design for Multiple Aircraft Conflict Resolution," in Proceedings of the American Control Conference, Chicago (IL), 2000, pp. 672-676.

[16] L. Pallottino, E. M. Feron, and A. Bicchi, "Conflict Resolution Problems for Air Traffic Management Systems Solved With Mixed Integer Programming," IEEE Transactions on Intelligent Transportation Systems, vol. 3, no. 1, pp. 3-11, Mar. 2002.

[17] D. J. Wing, R. A. Vivona, and D. A. Roscoe, "Airborne Tactical IntentBased Conflict Resolution Capability," in 9th AIAA Aviation, Technology, Integration, and Operations, 2009.

[18] J. M. Hoekstra, R. N. H. W. van Gent, and J. Groeneweg, "Airborne Separation Assurance Validation With Multiple Humans-In-The-Loop," in Proceedings of the 5th USA - Europe ATM Seminar, 2003.

[19] A. R. Pritchett, "Human-Computer Interaction in Aerospace," in The Human-Computer Interaction Handbook: Fundamentals, Evolving Technologies and Emerging Applications. Mahwah, NJ, USA: Lawrence Erlbaum Associates, Inc., 2003, pp. 861-882.

[20] E. L. Wiener and R. E. Curry, "Flight-Deck Automation: Promises and Problems," Ergonomics, vol. 23, no. 10, pp. 995-1011, 1980.

[21] L. Bainbridge, "Ironies of Automation," in New Technology and Human Error, J. Rasmussen, Ed. John Wiley and Sons, 1987.

[22] M. R. Endsley, "Automation and Situation Awareness," in Automation and Human Performance: Therory and Applications, R. Parasuraman and M. Mouloua, Eds. Hillsdale, NJ: Erlbaum Associates, 1996, pp. $163-181$.

[23] R. Parasuraman and V. Riley, "Humans and Automation: Use, Misuse, Disuse, Abuse," Human Factors, vol. 39, no. 2, pp. 230-253, 1997.

[24] M. R. Endsley, B. Bolte, and D. G. Jones, Designing for situation awareness: an approach to user centered design. Taylor and Francis, 2003.

[25] J. M. Flach, M. Mulder, and M. M. van Paassen, "The Concept of the Situation in Psychology," in A Cognitive Approach to Situation Awareness: Theory and Application, S. P. Banbury and S. Tremblay, Eds. Ashgate, 2004, pp. 42-60.

[26] K. J. Vicente, "Ecological Interface Design: Progress and Challenges," Human Factors, vol. 44, pp. 62-78, 2002.

[27] G. A. Jamieson, "Ecological Interface Design for Petrochemical Process Control: An Empirical Assessment," IEEE Transactions on Systems, Man, and Cybernetics, part A: Systems and Humans, vol. 37, no. 6, pp. 906-920, 2007.

[28] K. J. Vicente and J. Rasmussen, "Ecological Interface Design: Theoretical Foundations," IEEE Transactions on Systems, Man, and Cybernetics, vol. 22, no. 4, pp. 589-606, 1992.

[29] C. M. Burns and J. R. Hajdukiewicz, Ecological Interface Design. FL: Boca Raton: CRC Press LLC, 2004.

[30] J. J. Gibson, The Ecological Approach to Visual Perception. Houghton Mifflin, 1979.

[31] K. J. Vicente and J. Rasmussen, "The Ecology of Human-Machine Systems II: Mediating Direct-Perception in Complex Work Domains," Ecological Psychology, vol. 2, no. 3, pp. 207-249, 1990.

[32] J. Rasmussen, "Skills, Rules, Knowledge; Signals, Signs, Symbols, and Other Distinctions in Human Performance Models," IEEE Transactions on Systems, Man, and Cybernetics, vol. 13, pp. 257-266, 1983.

[33] J. J. Gibson, "The Theory of Affordances," Perceiving, Acting and Knowing: Toward an Ecological Psychology, pp. 67-82, 1977.

[34] M. H. J. Amelink, M. Mulder, M. M. van Paassen, and J. M. Flach, "Theoretical Foundations for a Total Energy-Based Perspective FlightPath Display," The International Journal of Aviation Psychology, vol. 15, pp. 205-231, 2005.

[35] C. Borst, H. C. H. Suijkerbuijk, M. Mulder, and M. M. van Paassen, "Ecological Interface Design for Terrain Awareness," International Journal of Aviation Psychology, vol. 16, no. 4, pp. 375-400, 2006.

[36] C. Borst, F. A. Sjer, M. Mulder, M. M. van Paassen, and J. A. Mulder, "Ecological Approach to Support Pilot Terrain Awareness After Total Engine Failure," Journal of Aircraft, vol. 45, no. 1, pp. 159-171, 2008.

[37] C. Borst, M. Mulder, and M. M. van Paassen, "Design and Simulator Evaluation of an Ecological Synthetic Vision Display," Journal of Guidance, Control and Dynamics, vol. 33, no. 5, pp. 1577-1591, 2010.

[38] S. B. J. van Dam, M. Mulder, and M. M. van Paassen, "Ecological Interface Design of a Tactical Airborne Separation Assistance Tool," 
IEEE Transactions on Systems, Man, and Cybernetics, part A: Systems and Humans, vol. 38, no. 6, pp. 1221-1233, 2008.

[39] F. M. Heylen, S. B. J. van Dam, M. Mulder, and M. M. van Paassen, "Design and Evaluation of a Vertical Separation Assistance Display," in AIAA Guidance, Navigation, and Control Conference and Exhibit, Honolulu (HI), 2008.

[40] J. Rasmussen, "The Role of Hierarchical Knowledge Representation in Decision Making and System Management," IEEE Transactions on Systems, Man, and Cybernetics, vol. 15, no. 2, pp. 234-243, 1985.

[41] G. Lintern, T. Waite, and D. A. Talleur, "Functional Interface Design for the Modern Aircraft Cockpit," The International Journal of Aviation Psychology, vol. 9, no. 3, pp. 225-240, 1999.

[42] M. M. van Paassen, "Functions of Space and Travellers," in Proceedings of 18th European Annual Conference on Human Decision Making and Manual Control. Faculty of Aerospace Engineering, Delft University of Technology, 1999.

[43] Radio Technical Commission for Aeronautics, "Airborne Conflict Management: Application Description V2.5," Federal Aviation Authorities, Tech. Rep. RTCA SC-186, 2002.

[44] A. M. Bisantz and K. J. Vicente, "Making the Abstraction Hierarchy Concrete," International Journal of Human-Computer Studies, vol. 40, pp. 83-117, 1994.

[45] M. M. van Paassen, M. H. J. Amelink, C. Borst, S. B. J. van Dam, and M. Mulder, "The Chicken, the Egg, the Workspace Analysis, and the Ecological Interface," in International Symposium on Aviation Psychology. Dayton, USA: Faculty of Aerospace Engineering, Delft University of Technology, 2007.

[46] Federal Aviation Administration and Eurocontrol, "Principles of Operation for the Use of Airborne Separation Assurance Systems," Federal Aviation Authorities - Eurocontrol, Tech. Rep. PO-ASAS-V7.1, 2001.

[47] S. B. J. van Dam, M. Mulder, and M. M. van Paassen, "The Use of Intent Information in an Airborne Self-Separation Assistance Display Design," in AIAA Guidance, Navigation, and Control Conference and Exhibit, 2009.

[48] Radio Technical Commission for Aeronautics, "Minimal Operational Performance Standards for Traffic Alert and Collision Avoidance System 2 (TCAS2) Airborne Equipment," Federal Aviation Authorities, Tech. Rep., 2002.

[49] — , "Final Report of the RTCA Board of Directors' Select Committee on Free Flight," RTCA, Tech. Rep., 1995.

[50] J. M. Hoekstra, "Designing for Safety: The Free Flight Air Traffic Management Concept," Ph.D. dissertation, Delft University of Technology, The Netherlands, 2001.

[51] C. D. Wickens, "The When and How of Using 2-D and 3-D Displays for Operational Tasks," in Proceedings of the Human Factors and Ergonomics Society, 2000, pp. 403-406.

[52] A. L. Alexander, C. D. Wickens, and D. H. Merwin, "Perspective and Coplanar Cockpit Displays of Traffic Information: Implications for Maneuver Choice, Flight Safety, and Mental Workload,' The International Journal of Aviation Psychology, vol. 15, pp. 1-21, 2005.

[53] M. W. McGreevy and S. R. Ellis, "The Effect of Perspective Geometry on Judged Direction in Spatial Information Instruments," Human Factors, vol. 28, no. 4, pp. 439-456, 1986.

[54] M. L. Bolton and E. J. Bass, "Using Relative Position and Temporal Judgments to Identify Biases in Spatial Awareness for Synthetic Vision Systems," The International Journal of Aviation Psychology, vol. 18, no. 2, pp. 183-206, 2008.

[55] R. Appleton, M. Mulder, and M. M. van Paassen, "Comparison of Two Interfaces for Supporting Pilots in Airborne Self-Separation Tasks," in AIAA Guidance, Navigation, and Control Conference and Exhibit. Keystone (CO), USA: AIAA, 2006.

[56] S. N. Roscoe, L. Corl, and R. S. Jensen, "Flight Display Dynamics Revisited," Human Factors, vol. 23, no. 3, pp. 341-353, Jun. 1981.

[57] D. J. Gates, E. A. Gates, M. Westcott, and N. L. Fulton, "Stereo Projections of Miss Distance in Some New Cockpit Display Formats," Journal of Aircraft, vol. 45, no. 5, pp. 1725-1735, 2008.

[58] R. A. Paielli, "Modeling Maneuver Dynamics in Air Traffic Conflict Resolution," Journal of Guidance Control and Dynamics, vol. 26, no. 3, pp. 407-415, May 2003.

[59] S. B. J. van Dam, M. Mulder, and M. M. van Paassen, "Airborne Self-Separation Display with Turn Dynamics and Intruder IntentInformation," in IEEE International Conference on Systems, Man and Cybernetics. Montreal, Canada: IEEE, 2007.

[60] M. R. Endsley, "Toward a Theory of Situation Awareness in Dynamic Systems," Human Factors, vol. 37, no. 1, pp. 32-64, 1995.
[61] K. Christoffersen, C. N. Hunter, and K. J. Vicente, “A Longitudinal Study of the Effects of Ecological Interface Design on Deep Knowledge," International Journal of Human-Computer Studies, vol. 48, pp. 729-762, 1998.

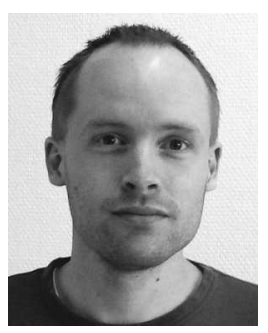

Joost Ellerbroek received the M.Sc. degree in aerospace engineering from the Delft University of Technology, Delft, The Netherlands, in 2007, where he is currently working toward the Ph.D. degree. His $\mathrm{Ph} . \mathrm{D}$. work concentrates on the design and validation of an interface that supports interaction with airborne separation automation. The research presented in this paper is part of his thesis.

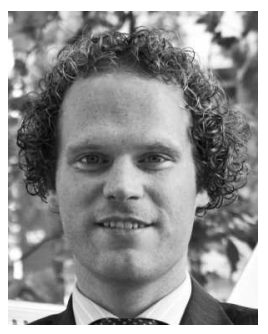

Mark Visser received the M.Sc. degree (cum laude) in aerospace engineering from the Delft University of Technology, Delft, The Netherlands, in 2009. His main interests are interface design, human-machine interaction and air traffic management. He graduated within the control and simulation division on his thesis entitled "Analysis and Design of a FourDimensional Separation Assistance Interface". The results of his work are incorporated in this paper.

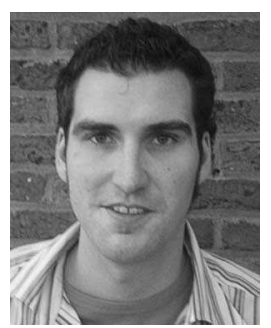

Stijn B. J. van Dam received the M.Sc. degree in aerospace engineering from the Delft University of Technology, Delft, The Netherlands, in 2004. He is currently working toward obtaining the Ph.D. degree. His Ph.D. work concentrates on the design and validation of an ecological self-separation assistance display in cruise flight. His research includes the design of ecological human-machine interfaces that support for vehicle control and traffic management in the aviation and maritime domains.

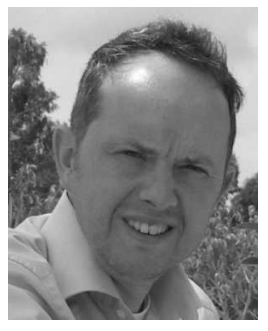

Max Mulder received the M.Sc. and Ph.D. degrees (cum laude) in aerospace engineering from the Delft University of Technology, Delft, The Netherlands, in 1992 and 1999, respectively, for his work on the cybernetics of tunnel-in-the-sky displays. He is currently Full Professor and Head of the Control and Simulation Division, Faculty of Aerospace Engineering, Delft University of Technology. His research interests include cybernetics and its use in modeling human perception and performance, and cognitive systems engineering and its application in the design of "ecological" human-machine interfaces. 


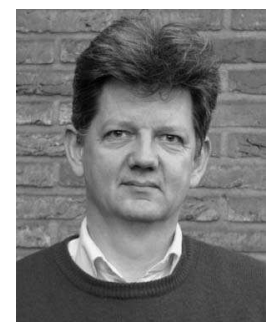

René M. M. van Paassen received the M.Sc. degree (cum laude) from the Delft University of Technology, Delft, The Netherlands, in 1988, where he also received the Ph.D. degree in 1994, with a thesis about a neuromuscular system of the pilots arm, for studying manual control of aircraft.

After this, he spent two years as a Brite/EuRam Research Fellow with the University of Kassel, Kassel, Germany, with Prof. Johannsen, where he worked on the development of alternative interfaces for process control based on functional models of the process. During a subsequent stay with the Technical University of Denmark, he put the practical experience gained in Kassel to use in theoretical work on multilevel flow modeling. Currently, he is with the Faculty of Aerospace Engineering, Delft University of Technology, working on aircraft simulation and human-machine interaction. 